\title{
SARCOPENIA E ESTADO NUTRICIONAL \\ DE IDOSOS RESIDENTES \\ EM UMA COMUNIDADE DO SUL DO BRASIL
}

\author{
Nathalia Cardoso de Oliveira ${ }^{1}$ \\ Fernanda Miraglia² \\ Fernanda Simas Moretto Tadini ${ }^{3}$ \\ Lidiane Isabel Filippin ${ }^{4}$
}

resumo

Introdução: Atualmente, a sarcopenia tem sido considerada como importante fator de risco para a redução da mobilidade, aumento no número de quedas e fraturas; e, é comumente associada com um alto risco de hospitalização, institucionalização, dependência, piora da qualidade de vida e mortalidade. Objetivo: verificar a prevalência de sarcopenia e o estado nutricional em idosos de um município do

\footnotetext{
1 Graduada em Fisioterapia pela Universidade La Salle Canoas - RS. E-mail: nati.cardoso.oliveira@ hotmail.com.

2 Graduada em Nutrição. Doutora em Saúde da Criança e do Adolescente. Docente do curso de Nutrição e do Programa de Pós-Graduação em Saúde e Desenvolvimento Humano da Universidade La Salle Canoas - RS. E-mail: fernandamiraglianutri@gmail.com.

3 Graduada em Nutrição pela Universidade La Salle Canoas - RS. E-mail: fernanda.simas95@ gmail.com.

4 Graduada em Fisioterapia. Doutora em Ciências Médicas. Docente do curso de Fisioterapia e do Programa de Pós-Graduação em Saúde e Desenvolvimento Humano da Universidade La Salle Canoas - RS. E-mail: lidiane.filippin@unilasalle.edu.br.
} 
Sul do Brasil. Métodos: trata-se de um estudo transversal de base populacional, realizado com idosos do Sul do Brasil. A sarcopenia foi determinada de acordo com o critério European Working Group on Sarcopenia in Older People (EWGSOP2). O estado nutricional foi avaliado pelo Questionário Mini Avaliação Nutricional (MAN). Aspectos socioeconômicos, demográficos e de desempenho físico foram descritos. Resultados: o estudo contou com uma amostra de 327 idosos; destes, 52,6\% apresentaram provável sarcopenia, 23,7\% obtiveram confirmação do diagnóstico de sarcopenia e 20,7\% apresentavam sarcopenia severa de acordo com os critérios de EWGSOP2. Na avaliação do estado nutricional pela MAN, todos os idosos foram considerados com estado nutricional normais, porém 34,3\% dos prováveis sarcopênicos apresentavam excesso de peso, enquanto 17,6\% idosos apresentaram risco de desnutrição e 2\% desnutridos. Conclusão: os dados apontam uma alta prevalência de idosos com provável sarcopenia (baixa força muscular) e excesso de peso (aumento da circunferência da cintura e índice de massa corporal), sugerindo um desequilíbrio energético nutricional desses idosos.

palavras-chave

Idoso. Sarcopenia. Estado Nutricional.

\section{Introdução}

O envelhecimento populacional tem aumentado e, paralelamente, emerge a necessidade do entendimento sobre as alterações físico-funcionais que acompanham esse fenômeno (WANG et al., 2013). Nas últimas décadas, vem sendo observado o grande avanço tecnológico, que é acompanhado de pesquisas investigando as possíveis teorias relacionadas ao envelhecimento e boas práticas relacionadas à saúde (WANG et al., 2015; IBGE, 2010). O envelhecimento, ou senescência, é um processo não patológico de deterioração de um organismo (CIOSAK et al., 2011), o qual impacta sobre o cuidado com a pessoa idosa. A Organização Mundial de Saúde (OMS) estima que, em 2050, tenha mais de dois bilhões de pessoas com idade superior a 60 anos em todo o mundo (OMS, 2005). Desses, 80\% viverão em países em desenvolvimento, incluindo o Brasil. Estima-se que, em 2025, o Brasil se torne o sexto país com maior percentual populacional de idosos no mundo (ZAGO, 2010). Com a expectativa de vida prolongada surge acréscimo de doenças crônicas, deficiências físicas, cognitivas 
e/ou nutricionais levando ao aparecimento de síndromes geriátricas, como a sarcopenia (FERREIRA et al., 2010; LI; HEBER, 2012).

A sarcopenia é definida como uma síndrome geriátrica, caracterizada pela perda progressiva da força muscular, massa muscular e/ou desempenho físico (CRUZ-JENTOFT et al., 2019) e, frequentemente, está associada ao aumento da gordura visceral (ZAMBONI et al., 2008). A perda de massa e de força muscular é uma variável clínica pontual e relevante, no entanto, ainda é um problema clínico subestimado, especialmente nos idosos mais velhos. Com o avanço da idade, há perda anual de 1 a $2 \%$ na massa muscular e de 1,5 a $5 \%$ na força muscular (HUGHES et al., 2002). A diminuição da força e da potência do músculo pode influenciar na autonomia (capacidade funcional) e no bem-estar dos longevos. A sarcopenia, atualmente, tem sido considerada importante fator de risco para a redução da mobilidade, maior número de quedas e fraturas, e está intimamente ligada com taxas elevadas de internações hospitalares, institucionalizações, dependência, piora na qualidade de vida e mortalidade (KINUGAWA et al., 2015; NARUMI et al., 2015; SERGI et al., 2016).

Um novo conceito de obesidade sarcopênica tem sido discutido e refere-se à sarcopenia associada ao aumento da gordura corporal (BAUMGARTNER, 2000; CHOI, 2016; ZAMBONI et al., 2008). As avaliações recentes destacaram o impacto da obesidade sarcopênica nos resultados gerais de saúde e no risco metabólico (CHOI, 2013; KOHARA, 2014; STENHOLM et al., 2008; WATERS; BAUMGARTNER, 2011; PRADO et al., 2012). O aparecimento e progressão da sarcopenia são multidimensionais, envolve inatividade física, alteração neuroendócrina, deterioração neuromuscular e risco nutricional (HOUSTON et al., 2008).

Alimentação adequada em combinação com o exercício físico são vistos como pilares importantes para o tratamento e prevenção da sarcopenia, juntamente com uma ingestão adequada de proteínas e vitamina $\mathrm{D}$, atualmente recomendados pelos profissionais da área da saúde (MORLEY et al., 2010; SCOTT et al., 2010). Além da deficiência de proteínas e da vitamina $D$, as vitaminas do complexo B, antioxidantes, ácidos graxos e ômega 3 foram relacionados como determinantes da sarcopenia (ROSENBERG, 1997).

Tendo em vista o impacto negativo dessas condições (sarcopenia e obesidade) diante da autonomia, qualidade de vida e risco de morbidades do indivíduo torna-se importante avaliar o paciente idoso de forma interdisciplinar e multiprofissional. Incluindo avaliações físicas, funcionais, clínica e do estado nutricional. Diante do exposto, o objetivo do nosso estudo foi verificar a prevalência de prováveis sarcopênicos e o estado nutricional em idosos residentes de um município do Sul do Brasil. 
Estudo de caráter transversal, de base domiciliar com residentes na área urbana de um município da região Sul do Brasil. O estudo foi conduzido no município de Nova Santa Rita (RS), pois apresenta restrito conhecimento sobre as condições básicas de saúde de sua população. Segundo dados do Instituto Brasileiro de Geografia e Estatística (IBGE), foram estimadas em 2016 uma população de 26.086 habitantes e uma densidade demográfica de 119,7 habitantes $/ \mathrm{km}^{2}$ (IBGE, 2010). Entretanto, segundo dados do DATASUS, a cidade possui apenas duas sedes de Estratégias de Saúde da Família (ESF), mesmo contando com vasta área territorial e número de habitantes.

O estudo trata-se de uma amostra por conglomerados em dois estágios: unidade geográfica e domicílio, respectivamente. Os setores censitários do IBGE foram utilizados como unidades primárias de seleção e o domicílio foi à unidade amostral. Foram selecionados para este estudo por amostragem simples $40 \%$ dos residentes com 60 ou mais anos de idade de cada setor censitário selecionado. Os dados foram coletados no domicílio do entrevistado, entre os meses de junho de 2014 e dezembro de 2015, por uma equipe de coletores devidamente treinada. Foram excluídos indivíduos com debilidade clínica grave e acamados.

\subsection{Aspectos éticos}

A pesquisa na íntegra, da qual este estudo faz parte, foi submetida e recebeu a aprovação do Comitê de Ética em Pesquisa da Universidade La Salle (CAAE: 30236314.0.0000.5307). Todos os participantes, ao aceitar participar da pesquisa, assinaram o Termo de Consentimento Livre e Esclarecido, respeitando a Resolução 466/2012.

\subsection{Avaliações}

O risco de sarcopenia foi avaliado de acordo com os critérios do European Working Group on Sarcopenia in Older People 2 (EWGSOP2) (CRUZ-JENTOFT et al., 2019) através da força muscular mensurada através da força de preensão manual (Dinamômetro Camry $\left.{ }^{\circledR}\right)$ : mulheres $(<16 \mathrm{~kg})$ e homens $(<27 \mathrm{~kg})$. Neste 
estudo, os participantes foram categorizados em não sarcopênicos (saudáveis) e prováveis sarcopênicos (baixa força muscular).

Variáveis Sociodemográficas: foram incluídas: idade, sexo, raça (determinada pelo avaliador como branca, parda, indígena ou negra), ocupação (emprego formal, emprego informal, empreendedor, desempregado, aposentado, pensionista, autônomo), escolaridade (em anos), estado civil (casados/união estável ou divorciado, separados ou viúvos, renda familiar (em salários-mínimos) e nível de atividade física avaliada pelo IPAQ (muito ativo: 75 minutos de atividade vigorosas por semana; ativo: 150 minutos de atividade leve ou moderado por semana; sedentário).

Desempenho funcional: foram avaliadas: a) mobilidade e o equilíbrio através do teste Timed Up-and-Go (TUG) (WALL et al., 2000; BISCHOFF et al., 2003); b) força dos membros inferiores foi avaliada pelo teste de sentar e levantar de trinta segundos (RIKLI; JONES, 2013).

Estado Nutricional: foi avaliado a partir do questionário Mini-avaliação Nutricional (MAN). O MAN é constituído por 18 questões que se dividem em duas partes. Na primeira parte, estão contidas seis perguntas relativas à avaliação da ingestão alimentar, perda de peso nos últimos três meses, mobilidade, ocorrência de estresse psicológico ou doença aguda recente, alterações neuropsicológicas e índice de massa corporal (IMC). A segunda aborda questões acerca de medidas antropométricas, como circunferências de braço e panturrilha, investigação alimentar, com o número de refeições consumidas, ingestão de líquidos e de alimentos, autonomia para alimentar-se, avaliação global, com perguntas relacionadas ao estilo de vida e medicamentos utilizados e auto avaliação relativa à saúde e nutrição do idoso (VELLAS et al., 1999; TYROVOLAS et al., 2016). Os idosos então classificados em três categorias conforme a pontuação: de 24 a 30 pontos: estado nutricional normal; de 17 a 23,5 pontos: sob risco de desnutrição; abaixo de 17 pontos: desnutrição.

Medidas antropométricas: as medidas antropométricas mensuradas foram massa e estatura para cálculo do índice de massa corporal (IMC), circunferência da cintura (CC). A obesidade central foi definida como uma CC $>102 \mathrm{~cm}$ para homens e $>88 \mathrm{~cm}$ para mulheres (WHO, 2008). A massa muscular total (MMT) foi mensurada através da equação preditiva de massa muscular esquelética (MME) proposta por Lee et al. (2000):

$$
\operatorname{MME}(\mathrm{kg})=(0,244 * \mathrm{MC})+(7,8 * \mathrm{EST})+(6,6 \text { * sexo })-(0,098 \text { * idade })+(\text { etnia }-3,3)
$$


O índice de massa muscular total (IMMT) foi calculado pela divisão da MMT pela estatura ao quadrado $\left(\mathrm{IMMT}=\mathrm{MMT} / \mathrm{estatura}^{2}\right)$ cujos valores de referência adotados para baixa quantidade muscular foram $<6,0 \mathrm{~kg} / \mathrm{m}^{2}$ e para homens $<7,0 \mathrm{~kg} / \mathrm{m}^{2}$ (GOULD et al., 2014).

\subsection{Procedimentos estatísticos}

Para análise estatística, foi utilizado medidas descritivas de médias, desvios padrão e porcentagem para a descrição das características sociodemográficas, estado geral de saúde e nutricional. As diferenças entre os grupos (não sarcopênicos e provável sarcopênicos) foram analisadas a partir do teste do qui-quadrado $\left(\chi^{2}\right)$ para variáveis categóricas e teste $t$ para amostras independes para variáveis simétricas e contínuas. O nível de significância adotado no presente estudo foi um valor de $p$ menor ou igual a 0,05 . Os dados foram analisados no The Statistical Package for Social Sciences para Windows (SPSS 22.0, 2013, SPSS, Inc., Chicago, IL).

\section{Resultados}

Participaram deste estudo 327 sujeitos, destes 52,6\% apresentaram provável sarcopenia. Dentre os indivíduos com provável sarcopenia ( $n=173), 23,7 \%$ obtiveram confirmação do diagnóstico de sarcopenia (n=78) e, 20,7\% apresentavam sarcopenia severa $(\mathrm{n}=68)$ de acordo com os critérios de EWGSOP2. As características sociodemográficas de todos os indivíduos estão apresentadas na Tabela 1.

Na avaliação do estado nutricional pela MAN, todos os idosos foram considerados com estado nutricional normal. Somente 17,6\% dos indivíduos com provável sarcopenia estavam sob risco de desnutrição e 2,0\% foram considerados desnutridos. Por outro lado, a maioria dos pacientes com provável sarcopenia apresenta excesso de peso $(34,3 \%)$ quando comparado com os indivíduos saudáveis $(\mathrm{p}<0,05)$. A CC confirma a obesidade central em ambos os sexos. Quando o IMC estratificado por sexo é analisado, as mulheres apresentam IMC significativamente maior quando comparado às mulheres saudáveis $(p<0,05)$. Essa diferença não é observada nos homens (prováveis sarcopênicos). O IMMT dos homens com provável sarcopenia não apresenta diferença significativa quando comparado ao IMMT dos indivíduos saudáveis. Entretanto, o IMMT das mulheres é significativamente menor quando comparado às mulheres saudáveis, mesmo com o excesso de peso (Tabela 2). 
Tabela 1 - Caraterísticas sociodemográficas e desempenho funcional da população do estudo EPES com diagnóstico de sarcopenia pelos critérios do EWGSOP2, Rio Grande do Sul, Brasil $(n=327)$

\begin{tabular}{|c|c|c|}
\hline Variáveis & $\begin{array}{l}\text { Não sarcopenia } \\
(47,4 \% ; n=156)\end{array}$ & $\begin{array}{l}\text { Provável } \\
\text { sarcopenia } \\
(52,6 \% ; n=173)\end{array}$ \\
\hline Idade (anos) & $67,7 \pm 5,41$ & $67,8 \pm 5,70$ \\
\hline Sexo (feminino) $[\%,(n)]$ & $53,5(83)$ & $66,3(114)$ \\
\hline Raça (caucasiana) [\%, (n)] & $87,7(136)$ & $89,0(153)$ \\
\hline Ocupação (aposentado) [\%, (n)] & $74,2(105)$ & $69,2(106)$ \\
\hline Escolaridade (anos) & $3,34 \pm 1,42$ & $3,23 \pm 1,63$ \\
\hline Estado civil (casado) [\%, (n)] & $62,0(80)$ & $52,7(69)$ \\
\hline Renda familiar (< 3 salários-mínimos) [\%, (n)] & $82,2(106)$ & $86,3(113)$ \\
\hline IPAQ (ativo) & $26,4(84)$ & $31,4(100)$ \\
\hline TUG (segundos) & $9,70 \pm 4,13$ & $9,15 \pm 2,88$ \\
\hline Força de membros inferiores (rep) & $10,0 \pm 2,86$ & $10,57 \pm 3,43$ \\
\hline
\end{tabular}

Fonte: Elaborada pelas autoras.

Nota: dados contínuos apresentados em média \pm desvio padrão; e dados categóricos apresentados em porcentagem e (valor absoluto).

Raça: (determinada pelo avaliador como branca, parda, indígena ou negra); Estado civil (casado ou solteiro); Renda familiar (estratificada em salários-mínimos); TUG: Timed Up-and-Go;

rep: repetições.

Tabela 2 - Características nutricionais de população com provável sarcopenia pelos critérios do EWGSOP2, Rio Grande do Sul, Brasil (n=327)

\begin{tabular}{lll}
\hline Variáveis & $\begin{array}{l}\text { Não sarcopênicos } \\
\mathbf{( 4 7 , 4 \% ; n = 1 5 6 )}\end{array}$ & $\begin{array}{l}\text { Provável sarcopenia } \\
\mathbf{( 5 2 , 6 \% ; n = 1 7 3 )}\end{array}$ \\
\hline MAN & $23,7 \pm 2,69$ & $23,8 \pm 2,93$ \\
Risco nutricional & & \\
Desnutrido & $2,0(5)$ & $2,0(5)$ \\
Sob risco de desnutrição & $20,9(51)$ & $17,6(43)$ \\
Normal & $25,0(61)$ & $32,4(79)$ \\
\hline
\end{tabular}




\begin{tabular}{|c|c|c|}
\hline Variáveis & $\begin{array}{l}\text { Não sarcopênicos } \\
(47,4 \% ; n=156)\end{array}$ & $\begin{array}{l}\text { Provável sarcopenia } \\
(52,6 \% ; n=173)\end{array}$ \\
\hline IMC & $27,97 \pm 5,31$ & $29,54 \pm 41 \pm 6,48^{*}$ \\
\hline Homens & $28,54 \pm 511$ & $28,90 \pm 6,65$ \\
\hline Mulheres & $27,49 \pm 5,46$ & $29,87 \pm 6,39^{*}$ \\
\hline \multicolumn{3}{|l|}{ IMC } \\
\hline Magreza & $5,2(17)$ & $11(3,4)$ \\
\hline Eutrófico & $15,9(52)$ & $15,0(49)$ \\
\hline Excesso de peso & $26,3(86)$ & $34,3(112)^{\ddagger}$ \\
\hline \multicolumn{3}{|l|}{$\mathrm{CC}(\mathrm{cm})$} \\
\hline Homens & $98,95 \pm 11,08$ & $102,17 \pm 12,32$ \\
\hline Mulheres & $97,77 \pm 14,12$ & $95,08 \pm 13,50$ \\
\hline \multicolumn{3}{|l|}{ MMT (kg) } \\
\hline Homens & $28,53 \pm 4,23$ & $29,86 \pm 5,34$ \\
\hline Mulheres & $20,25 \pm 4,54$ & $19,10 \pm 4,31$ \\
\hline \multicolumn{3}{|l|}{ IMMT (kg/m²) } \\
\hline Homens & $9,95 \pm 1,44$ & $10,46 \pm 1,44$ \\
\hline Mulheres & $8,39 \pm 1,82$ & $7,90 \pm 1,45^{*}$ \\
\hline
\end{tabular}

Fonte: Elaborada pelas autoras.

Nota: dados apresentados em média \pm desvio padrão e porcentagem.

*teste t para amostras independentes, $p<0,05$;

*teste de $\chi^{2}, p<0,05$;

MAN: mini avaliação nutricional; IMC: índice de massa corporal; CC: circunferência da cintura; cm: centímetros; MMT: Massa muscular total; kg: quilograma; IMMT: Índice de massa muscular total; $\mathrm{kg} / \mathrm{m}^{2}$ : quilograma por metro ao quadrado.

\section{Discussão}

Os principais achados deste estudo demonstram que existe uma alta prevalência de idosos com provável sarcopenia observada pela baixa força muscular e obesidade mensurada através do IMC e CC. Tais resultados merecem atenção, visto que sarcopenia e obesidade estão associados a desordens 
energético-nutricionais e têm sido apontadas como um aspecto negativo da saúde do idoso, com risco aumentado de doenças e mortalidade (ALLISON et al., 2002), diminuindo sua capacidade funcional e qualidade de vida (WANNAMETHEE; ATKINS, 2015). Portanto, a identificação de indivíduos com provável sarcopenia e risco de excesso de peso, contribui na adoção de medidas de prevenção, melhorando a qualidade de vida e capacidade funcional desta população.

A prevalência de sarcopenia evidenciada neste estudo (23,7\%) foi discretamente mais elevada às encontradas em outros estudos nas Américas, Europa e Ásia (TYROVOLAS et al., 2016). No entanto, deve-se considerar que o presente estudo utilizou no novo consenso proposto pelo EWGSOP2, o qual reduz o cut-offe, consequentemente, mais indivíduos tornam-se verdadeiros positivos. Em recente revisão sistemática sobre a prevalência de sarcopenia ao redor do mundo utilizando três distintos consensos, foi observado que a prevalência em ambos os sexos está em torno de 10\% (IC95\%: 8 - 13\%) (SHAFIEE et al., 2017). No Brasil, estudo realizado em idosas residentes na comunidade na Bahia demonstrou uma prevalência de 17,8\% de sarcopenia (DUTRA et al., 2015). Em idosos no estado de São Paulo, a prevalência foi de 15,4\% (ALEXANDRE et al., 2014).Na região Sul, a prevalência encontrada em idosos residentes na comunidade na cidade de Pelotas no Rio Grande do Sul foi de 13,9\% (BARBOSA-SILVA et al., 2016). Estudo semelhante realizado apenas com idosas residentes na comunidade em Santa Maria (RS) apresentou prevalência de sarcopenia de 17,2\% (SALAME et al., 2015). Já no estudo de Vetrano et al. (2014), a prevalência de sarcopenia foi alta, chegando a $28 \%$ em indivíduos hospitalizados com mais de 65 anos de idade. Smoliner, Sieber e Wirth (2014) investigaram pacientes idosos admitidos em cuidados geriátricos agudos por diferentes problemas de saúde. Foi observado que a prevalência da síndrome era $25,3 \%$, porém um número maior de pacientes apresentava sarcopenia severa (18,7\%). Os estudos apontam que as maiores taxas de sarcopenia em pacientes hospitalizados deve-se ao fato da maior presença de comorbidades (diabetes, insuficiência cardíaca, doença pulmonar obstrutiva, demência) e condições clínicas piores (maior número de medicações, dor crônica, úlceras de pressão, diarreia crônica).

O presente estudo demonstrou uma alta proporção de indivíduos com provável sarcopenia $(52,6 \%)$ avaliado pela redução da força muscular conforme os novos critérios propostos pelo EWGSOP2. Essa foi uma modificação fundamental na revisão do EWGSOP2, pois permite intervenções preventivas nessa população somente com a redução de força muscular. Estudos têm demonstrado que a redução da força muscular (dinapenia) foi considerada fator de risco independente para morte em idosos ( $\mathrm{HR}=2,04$; CI95\%: 1,24-3) 
(ALEXANDRE et al., 2014; LI et al., 2018; BOHANNON, 2015). No consenso anterior (CRUZ-JENTOFT et al., 2010), era imperativo a imagem muscular para avaliar a quantidade muscular (DXA ou bioimpedância) dificultando a avaliação dos idosos na comunidade e na atenção secundária. Dessa maneira, existia dificuldade do diagnóstico precoce comprometendo de maneira negativa a qualidade de vida desses idosos, afetando a funcionalidade, o que pode dificultar o desempenho das atividades de vida diária, e, como consequência, aumentará a incidência de quedas e dependência funcional. Para Gomes et al. (2009), a sarcopenia pode ser uma das principais causas de quedas nos idosos. Com uma amostra de 72 idosos (25 homens e 47 mulheres), com média de idade de 76,3 $\pm 8,3$ anos, demonstraram que o risco de queda é diretamente proporcional à faixa etária, assim como ao número de medicações consumidas pelos pacientes. A polifarmácia e a sarcopenia em indivíduos idosos podem superestimar o risco de quedas, uma vez que algumas medicações podem afetar o equilíbrio do idoso. Por isso, esse viés deve ser avaliado paralelamente à presença da sarcopenia.

O envelhecimento, embora seja um processo natural e esperado, leva o corpo a diversas alterações anatômicas e funcionais, com profundas repercussões nas condições de saúde e nutrição do idoso. A associação com a polifarmácia influencia na ingestão de alimentos, na digestão e absorção de diversos nutrientes. Esse quadro pode comprometer o estado geral de saúde e, especialmente, o estado nutricional do idoso.

Em nosso estudo, em idosos com provável sarcopenia residentes na comunidade, a prevalência de desnutrição foi baixa $(2,0 \%)$, a maior parte, $32,4 \%$ apresentam estado nutricional normal. Somado, 34,3\% da amostra apresentou excesso de peso (IMC $>27 \mathrm{Kg} / \mathrm{m}^{2}$ ) e aumento da CC. Quando os idosos com provável sarcopenia foram estratificados pelo sexo, as mulheres apresentaram IMC e CC mais elevado quando comparada às idosas saudáveis. Todavia, o IMMT das prováveis sarcopênicas foi significativamente menor quando comparado às idosas saudáveis $(7,90 \pm 1,45$ vs. $8,39 \pm 1,82)$. Esses dados sugerem que as mulheres idosas residentes na comunidade podem apresentar obesidade sarcopênica.

As alterações na composição corporal relacionadas à idade (ou seja, declínio da massa muscular e aumento da massa gorda) parecem estar fortemente conectadas entre si do ponto de vista patogenético. A perda de massa muscular induz um declínio de $2 \%$ a 3\% na taxa metabólica basal por década após os 20 anos de idade e um declínio de $4 \%$ por década após os 50 anos. Isso pode levar a um aumento do risco de ganho de peso, caracterizado principalmente pelo acúmulo de gordura abdominal, com menor atividade física realizada 
em segundo plano (NAIR, 2005; ZAMBONI et al., 2008; BUCH et al., 2016). Alternativamente, a gordura visceral induz inflamação subclínica que pode contribuir para o desenvolvimento de sarcopenia (TILG; MOSCHEN, 2006; GREGOR; HOTAMISLIGIL, 2011). Portanto, é possível hipotetizar um papel das células adiposas e macrófagos na patogênese da sarcopenia.

Em um estudo longitudinal com adultos coreanos (Korean Sarcopenic Obesity Study - KSOS) foi observado que obesidade visceral estava associada à perda futura de massa muscular esquelética. Os autores demonstraram uma correlação inversa entre a obesidade visceral no baseline e a massa muscular esquelética ao final do seguimento (28 meses) $(\mathrm{r}=-0,20, \mathrm{p}<0,001)$.

Esse estudo apresenta algumas limitações. A primeira limitação foi não realizar uma avaliação da ingestão alimentar desta população, porém foi possível inferir através da composição corporal (CC e IMC) que estes indivíduos se apresentam obesos e com deposição de gordura abdominal demonstrando um possível desbalanço energético e nutricional. A segunda limitação é não conseguir concluir a provável obesidade sarcopênica apresentada pelas mulheres idosas devido aos critérios propostos atualmente na literatura (avaliação de massa gorda e livre de gordura por exame de imagem - DXA), impossibilitando de realizar no domicílio ou de rastrear esses indivíduos.

\section{Conclusão}

Finalmente, a sarcopenia e o excesso de peso devem ser vistas como um problema de saúde pública, sobretudo por estar associada ao aumento de gastos econômicos nos sistemas de saúde na maioria dos países. Em relação à prevenção, o foco é promover a saúde através da prática de exercício físico e de uma adequada ingestão de nutrientes, pois estes auxiliarão na precaução da perda de massa óssea e muscular. Também é de extrema relevância conscientizar a população a tornar-se participativa, por intermédio do controle social, o que somente será alcançado com a elaboração e implementação de novas políticas de educação primária na saúde. Assim, o estudo sugere avaliação periódica e regular da força muscular em idosos residentes na comunidade para implementação de medidas preventivas da sarcopenia. 


\title{
abstract
}

Introduction: Currently, sarcopenia has been considered an important risk factor for the reduction of mobility, an increase in the number of falls and fractures, and is commonly associated with a high risk of hospitalization, institutionalization, dependence, worsening of quality of life and mortality. Purpose: to verify the prevalence of sarcopenia and nutritional status in older adults in a city in the South of Brazil. Methods: this is a cross-sectional population-based study, carried out with older people in southern Brazil. Sarcopenia was determined according to the European Working Group on Sarcopenia in Older People (EWGSOP2) criteria. Nutritional status was assessed using the Mini Nutritional Assessment Questionnaire (MAN). Socioeconomic and demographic aspects and physical performance were described. Results: the study included a sample of 327 older people, of whom $52.6 \%$ had probable sarcopenia, 23.7\% obtained confirmation of the diagnosis of sarcopenia and, 20.7\% had severe sarcopenia according to the EWGSOP2 criteria. In the assessment of nutritional status by MAN, all the older adults were considered to have a normal nutritional status, however, 34.3\% of the probable sarcopenics were overweight, while $17.6 \%$ were at risk of malnutrition and $2 \%$ were malnourished. Conclusion: the data point to a high prevalence of older people with probable sarcopenia (low muscle strength) and excess weight (increased waist circumference and body mass index), suggesting a nutritional energy imbalance in these older people.

keywords

Aged. Sarcopenia. Nutritional Status.

referências

\begin{abstract}
ALEXANDRE, Tiago da Silva et al. Prevalence and associated factors of sarcopenia among elderly in Brazil: findings from the SABE study. The Journal of Nutrition, Health \& Aging, New York, v. 18, n. 3, p. 284-290, 2014

ALLISON, David Bradley et al. Differential associations of body mass index and adiposity with all-cause mortality among men in the first and second National Health and Nutrition Examination Surveys (NHANES I and NHANES II) follow-up studies. International Journal of Obesity and Related Metabolic Disorders, London, v. 26, n. 3, p. 410-416, Mar. 2002.
\end{abstract}


BARBOSA-SILVA, Thiago Gonzalez et al. Prevalence of sarcopenia among community-dwelling elderly of a medium-sized South American city: results of the COMO VAl? study. Journal of Cachexia, Sarcopenia and Muscle, Berlin, v. 7, n. 2, p. 136-143, 2016.

BAUMGARTNER, Richard. Body composition in healthy aging. Annals of the New York Academy of Sciences, New York, v. 904, n. 1, p. 437-448, May 2000.

BISCHOFF, Heike et al. Identifying a cut-off point for normal mobility: a comparison of the timed 'up and go' test in community-dwelling and institutionalized elderly women. Age and Ageing, United Kingdom, v. 32, n. 3, p. 315-320, May 2003.

BOHANNON, Richard. Muscle strength: clinical and prognostic value of hand-grip dynamometry. Current Opinion in Clinical Nutrition and Metabolic Care, United States, v. 18, n. 5, p. 465-470, Sept. 2015.

$\mathrm{BUCH}$, Assaf et al. Muscle function and fat content in relation to sarcopenia, obesity and frailty of old age: an overview. Experimental Gerontology, Netherlands, v. 76, p. 25-32, Apr. 2016.

$\mathrm{CHOl}$, Kyung Mook. Sarcopenia and sarcopenic obesity. Endocrinology and Metabolism, Seoul, v. 28, n. 2, p. 86-89, June 2013.

$\mathrm{CHOl}$, Kyung Mook. Sarcopenia and sarcopenic obesity. The Korean Journal of Internal Medicine, Seoul, v. 31, n. 6, p. 1054-1060, Nov. 2016.

CIOSAK, Suely Itsuko et al. Senescência e senilidade: novo paradigma na Atenção Básica de Saúde. Revista da Escola de Enfermagem da USP, São Paulo, v. 45, n. esp. 2, p. 1763-1768, 2011

CRUZ-JENTOFT, Alfonso et al. Sarcopenia: European consensus on definition and diagnosis - Report of the European Working Group on Sarcopenia in Older People. Age and Ageing, United Kingdom, v. 39, n. 4, p. 412-423, July 2010.

CRUZ-JENTOFT, Alfonso et al. Sarcopenia: revised European consensus on definition and diagnosis. European Working Group on Sarcopenia in Older People 2 (EWGSOP2). Age and Ageing, United Kingdom, v. 48, n. 1, p. 16-31, Jan. 2019.

DUTRA, Thaliany et al. Prevalência e fatores associados a sarcopenia em mulheres idosas residentes em comunidade. Revista Brasileira de Cineantropometria \& Desempenho Humano, Florianópolis, v. 17, n. 4, p. 460-471, jul./ago. 2015.

FERREIRA, Fabiane Ribeiro et al. Aging and urbanization: the neighborhood perception and functional performance of elderly persons in Belo Horizonte Metropolitan Area Brazil. Journal of Urban Health, United States, v. 87, n. 1, p. 54-66, Jan. 2010.

GOMES, Grace et al. Comparação entre idosos que sofreram quedas segundo desempenho físico e número de ocorrências. Revista Brasileira de Fisioterapia, São Carlos, v. 13, n. 5, p. 430-437, set./out. 2009.

GOULD, Haslinda et al. Total and appendicular lean mass reference ranges for Australian men and women: the Geelong osteoporosis study. Calcified Tissue International, United States, v. 94, n. 4, p. 363-372, Apr. 2014.

GREGOR, Margaret; HOTAMISLIGIL, Gökhan. Inflammatory mechanisms in obesity. Annual Review of Immunology, United States, v. 29, p. 415-445, 2011.

HOUSTON, Denise Kathryn et al. Dietary protein intake is associated with lean mass change in older, community-dwelling adults: The Health, Aging, and Body Composition (Health ABC) Study. American Journal of Clinical Nutrition, Bethesda, v. 87, n. 1, p. 150-155, Jan. 2008

HUGHES Virginia et al. Longitudinal changes in body composition in older men and women: role of body weight change and physical activity. American Journal of Clinical Nutrition, Bethesda, v. 76, n. 2, p. 473-481, Aug. 2002. 

2010. Rio de Janeiro: IBGE, 2010.

KINUGAWA, Shintaro et al. Skeletal muscle abnormalities in heart failure. International Heart Journal, Tokyo, v. 56, n. 5, p. 475-484, 2015.

KOHARA, Katsuhiko. Sarcopenic obesity in aging population: current status and future directions for research. Endocrine, Switzerland, v. 45, n. 1, p. 15-25, Feb. 2014.

LEE, Robert et al. Total-body skeletal muscle mass; development and cross-validation of anthropometric prediction models. American Journal of Clinical Nutrition, Bethesda, v. 72, n. 3, p. 796-803, Sept. 2000.

LI, Ran et al. Associations of muscle mass and strength with all-cause mortality among US older adults. Medicine \& Science in Sports \& Exercise, Indianapolis, v. 50, n. 3, p. 458-467, Mar. 2018.

LI, Zhaoping; HEBER, David. Sarcopenic obesity in the elderly and strategies for weight management. Nutrition Reviews, New York, v. 70, n. 1, p. 57-64, Jan. 2012.

MORLEY, John et al. Nutritional recommendations for the management of sarcopenia. Journal of the American Medical Directors Association, Hagerstown, v. 11, n. 6, p. 391-396, July 2010

NAIR, Sreekumaran. Aging muscle. American Journal of Clinical Nutrition, Bethesda, v. 81, n. 5, p. 953-963, May 2005.

NARUMI, Taro et al. Sarcopenia evaluated by fat-free mass index is an important prognostic factor in patients with chronic heart failure. European Journal of Internal Medicine, Amsterdam, v. 26, n. 2, p. 118-122, Mar. 2015.

ORGANIZAÇÃO MUNDIAL DA SAÚDE (OMS). Envelhecimento ativo: uma política de saúde. Tradução de Suzana Gontijo. Brasília, DF: Organização Pan-Americana da Saúde, 2005

PRADO, Carla et al. Sarcopenic obesity: a critical appraisal of the current evidence. Clinical Nutrition, Edinburgh, v. 31, n. 5, p. 583-601, Oct. 2012.

RIKLI, Roberta; JONES, Jessie. Development and validation of criterion-referenced clinically relevant fitness standards for maintaining physical independence in later years, Gerontologist, Washington, DC, v. 53, n. 2, p. 255-267, Apr. 2013.

ROSENBERG, Irwin. Sarcopenia: origins and clinical relevance. Journal of Nutrition, Philadelphia, v. 127, n. 5, p. 990S-991S, May 1997.

SALAME, Marcelo et al. Sarcopenia: avaliação de diferentes critérios diagnósticos e sua associação com a força muscular e capacidade funcional. Revista Brasileira de Geriatria e Gerontologia, Rio de Janeiro, v. 18, n. 2, p. 285-294, 2015.

SCOTT, David et al. Associations between dietary nutrient intake and muscle mass and strength in community-dwelling older adults: the Tasmanian Older Adult Cohort Study. Journal of the American Geriatrics Society, New York, v. 58, n. 11, p. 2129-2134, Nov. 2010

SERGl, Giuseppe et al. Imaging of sarcopenia. European Journal of Radiology, Germany, v. 85, n. 8, p. 1519-1524, Aug. 2016.

SHAFIEE, Gita et al. Prevalence of sarcopenia in the world: a systematic review and meta-analysis of general population studies. Journal of Diabetes \& Metabolic Disorders, United Kingdom, v. 16, n. 21, May 2017.

SMOLINER, Christine; SIEBER, Cornel; WIRT, Rainer. Prevalence of sarcopenia in geriatric hospitalized patients. Journal of the American Medical Directors Association, Hagerstown, v. 15, n. 4, p. 267-272, Apr. 2014. 
STENHOLM, Sari et al. Sarcopenic obesity: definition, cause and consequences. Current Opinion in Clinical Nutrition and Metabolic Care, United States, v. 11, n. 6, p. 693-700, Nov. 2008.

TILG, Herbert; MOSCHEN, Alexander. Adipocytokines: mediators linking adipose tissue, inflammation and immunity. Nature Reviews Immunology, United Kingdom, v. 6, n. 10, p. $772-783$, Oct. 2006

TYROVOLAS, Stefanos et al. Factors associated with skeletal muscle mass, sarcopenia, and sarcopenic obesity in older adults: a multi-continent study. Journal of Cachexia, Sarcopenia and Muscle, Berlin, v. 7, n. 3, p. 312-321, June 2016.

VELLAS, Bruno et al. The Mini Nutritional Assessment (MNA) and its use in grading the nutritional state of elderly patients. Nutrition, Burbank, v. 15, n. 2, p. 116-122, Feb. 1999.

VETRANO, Davide et al. Association of sarcopenia with short- and long-term mortality in older adults admitted to acute care wards: results from the CRIME study. Journal of Gerontology Series A: Biological Sciences and Medical Sciences, Washington, DC v. 69, n. 9, p. 1154-1161, Sept. 2014.

WALL, James et al. The Timed Get-Up-and-Go test revisited: measurement of the component tasks. Journal of Rehabilitation Research \& Development, Washington, DC, v. 37, n. 1, p. 109-113, Jan./Feb. 2000.

WANG, Rong et al. Incidence and effects of Polypharmacy on clinical outcome among patients aged 80+: a five-year follow-up study. PLoS One, San Francisco, v. 10, n. 11, e0142123, Nov. 2015.

WANG, Shi-Yi et al. Not just specific diseases: systematic review of the association of geriatric syndromes with hospitalization or nursing home admission. Archives of Gerontology and Geriatrics, Amsterdam, v. 57, n. 1, p. 16-26, July/Aug. 2013.

WANNAMETHEE, Goya; ATKINS, Janice. Muscle loss and obesity: the health implications of sarcopenia and sarcopenic obesity. Proceedings of the Nutrition Society, United Kingdom, v. 74, n. 4, p. 405-412, Nov. 2015.

WATERS, Debra; BAUMGARTNER, Richard. Sarcopenia and obesity. Clinics in Geriatric Medicine, United States, v. 27, n. 3, p. 401-421, Aug. 2011.

WORLD HEALTH ORGANIZATION (WHO). Waist circumference and waist-hip ratio: report of a WHO expert consultation. Geneva: World Health Organization, 2008.

ZAGO, Anderson Saranz. Exercício físico e o processo saúde-doença no envelhecimento. Revista Brasileira de Geriatria e Gerontologia, Rio de Janeiro, v. 13, n. 1 , p. $153-158,2010$.

ZAMBONI, Mauro et al. Sarcopenic obesity: a new category of obesity in the elderly. Nutrition, Metabolism and Cardiovascular Diseases, Italy, v. 18, n. 5, p. 388-395, June 2008.

Data de Submissão: 03/06/2019

Data de Aprovação: 10/04/2020 
